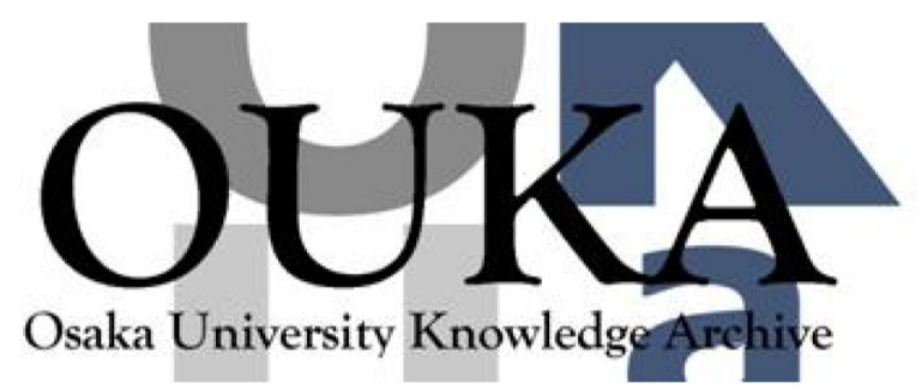

\begin{tabular}{|c|l|}
\hline Title & Triple point of Yukawa systems \\
\hline Author(s) & Hamaguchi, S.; Farouki, R. T.; Dubin, D. H. E. \\
\hline Citation & $\begin{array}{l}\text { Physical Review E - Statistical Physics, } \\
\text { Plasmas, F luids, and Re lated Interdisciplinary } \\
\text { Topics. 56(4) p. 4671-p.4682 }\end{array}$ \\
\hline Issue Date & $1997-10$ \\
\hline oaire:version & VoR \\
\hline URL & https://hdl. handle. net/11094/78497 \\
\hline rights & $\begin{array}{l}\text { Copyright (1997) by the American Physical } \\
\text { Society. }\end{array}$ \\
\hline Note & \\
\hline
\end{tabular}

Osaka University Knowledge Archive : OUKA

https://ir. Library. osaka-u. ac. jp/

Osaka University 


\title{
Triple point of Yukawa systems
}

\author{
S. Hamaguchi \\ IBM Thomas J. Watson Research Center, P.O. Box 218, Yorktown Heights, New York 10598 \\ R. T. Farouki \\ Department of Mechanical Engineering and Applied Mechanics, University of Michigan, Ann Arbor, Michigan 48109 \\ D. H. E. Dubin \\ Department of Physics, University of California at San Diego, La Jolla, California 92093
}

(Received 2 May 1997)

\begin{abstract}
The molecular dynamics simulations of Yukawa (i.e., screened-Coulomb) systems that were applied to the regime of weak screening in an earlier study [S. Hamaguchi, R. T. Farouki, and D. H. E. Dubin, J. Chem. Phys. 105, 7641 (1996)] are extended to the strong screening regime. Transition temperatures at the fluid-solid phase boundary and the solid-solid phase boundary are obtained as functions of the screening parameter $\kappa=a / \lambda_{D}$ (i.e., the ratio of the Wigner-Seitz radius $a$ to the Debye length $\lambda_{D}$ ). The resulting phase diagram also covers the triple point - the intersection of the fluid-solid and solid-solid phase boundaries-at $\kappa=4.28$ and $\Gamma=5.6$ $\times 10^{3}$, where $\Gamma$ is the ratio of the Coulomb potential energy to the kinetic energy per particle (i.e., $\Gamma$ $=Q^{2} / 4 \pi \epsilon_{0} a k T$, where $Q$ is the charge of each Yukawa particle and $T$ is the system temperature). Yukawa systems serve as models for plasmas and colloidal suspensions of charged particulates.
\end{abstract}

[S1063-651X(97)11310-1]

PACS number(s): 52.25.Vy, 64.60.-i, 82.70.Dd, 98.38.Cp

\section{INTRODUCTION}

Small charged "dust" grains are observed in a wide variety of plasma environments, ranging from the interstellar medium to gas discharges used in materials processing. Small particles immersed in a plasma typically acquire negative charges, due to the high mobility of plasma electrons. The Coulomb interactions between such particles are modified by their Debye sheaths, and the interparticle potential may be approximated by a Yukawa-type (screened Coulomb) pair potential [1-3] as given in Eq. (1) below.

Laboratory experiments have recently demonstrated that, when the interparticle potential energy exceeds the kinetic energy, particulates in plasmas may form crystalline structures (Coulomb crystals) [4-10]. Similar crystals have been observed in colloidal suspensions of charged particles [11]. As in our earlier report [1], we shall employ Yukawa systems as a model for plasma (or colloidal) suspensions of charged particles. In the present study, however, we extend our molecular dynamics (MD) simulations to the regime of strong screening of the Yukawa potential, and determine the conditions under which dust particles in a plasma will form Coulomb crystals.

We consider a system of identical particles of mass $m$ and charge $-Q=-Z e(Z \gg 1)$, immersed in a neutralizing background plasma. The inter-particle potential is assumed to be of the Yukawa type,

$$
\phi(r)=\frac{Q^{2}}{4 \pi \epsilon_{0} r} \exp \left(-k_{D} r\right)
$$

where $r$ denotes the radial distance between two particles. The Debye length $\lambda_{D}=k_{D}^{-1}$ of the background plasma is defined by

$$
\lambda_{D}=\left(\frac{q_{i}^{2} \overline{n_{i}}}{\varepsilon_{0} k T_{i}}+\frac{e^{2} \bar{n}_{e}}{\varepsilon_{0} k T_{e}}\right)^{-1 / 2},
$$

$q_{i}, \overline{n_{i}}$, and $T_{i}$ being the charge, mean density, and temperature of plasma ions, and $-e, \bar{n}_{e}$, and $T_{e}$ the corresponding quantities for plasma electrons. The thermodynamics of the Yukawa system can be characterized by two dimensionless parameters:

$$
\kappa=\frac{a}{\lambda_{D}} \quad \text { and } \quad \Gamma=\frac{Q^{2}}{4 \pi \epsilon_{0} a k T},
$$

where $a=(3 / 4 \pi n)^{1 / 3}$ is the Wigner-Seitz radius and $n$ is the particle number density. The Wigner-Seitz radius represents the mean interparticle distance, and $\Gamma$ is roughly the ratio of the (unscreened) Coulomb potential energy to the kinetic energy per particle.

In our earlier study [1] we focused on the regime of weak Debye screening, including the limit $\kappa \rightarrow 0$, i.e., the classical one-component plasma (OCP) system [12-18]. In our MD simulation method, long-range particle interactions are accurately accounted for over the entire range of $\kappa$, without introducing a cutoff radius for the pair potential. In this paper, we apply this MD method to more strongly screened Yukawa systems, up to $\kappa=5$, and compare the results with those of earlier MD and Monte Carlo (MC) simulations [1923] of Yukawa systems. These earlier simulations employed potential cutoffs, and are therefore applicable only to the regime $\kappa \gg 1$.

\section{EXCESS ENERGY AND FREE ENERGY}

In MD simulations, one can calculate the potential or "excess" energy $U$ of the model system in the simulation 
TABLE I. Madelung energies for bcc and fcc Yukawa lattices (for $\kappa \leqslant 1.0$, see Table III of Ref. [3]).

\begin{tabular}{ccc}
\hline \hline$\kappa$ & $E_{\mathrm{bcc}}$ & $E_{\mathrm{fcc}}$ \\
\hline 1.2 & -1.039292 & -1.039302 \\
1.4 & -1.088350 & -1.088374 \\
1.6 & -1.143352 & -1.143389 \\
1.8 & -1.203757 & -1.203803 \\
2.0 & -1.269026 & -1.269079 \\
2.2 & -1.338637 & -1.338694 \\
2.4 & -1.412096 & -1.412154 \\
2.6 & -1.488941 & -1.488998 \\
2.8 & -1.568750 & -1.568804 \\
3.0 & -1.651144 & -1.651194 \\
3.2 & -1.735781 & -1.735826 \\
3.4 & -1.822360 & -1.822400 \\
3.6 & -1.910618 & -1.910653 \\
3.8 & -2.000326 & -2.000356 \\
4.0 & -2.091283 & -2.091309 \\
4.2 & -2.183319 & -2.183341 \\
4.4 & -2.276286 & -2.276304 \\
4.6 & -2.370058 & -2.370072 \\
4.8 & -2.464525 & -2.464537 \\
5.0 & -2.559596 & -2.559606 \\
\hline
\end{tabular}

volume, under appropriate boundary conditions, for a given system temperature $T$. The method of calculating $U$ from MD is briefly discussed in the following section. In this section we shall derive the Helmholtz free energy $F$ in the simulation volume from a knowledge of $U$ as a function of the temperature $T$. We denote the internal energy and Helmholtz free energy per particle in units of $k T$ by

$$
u=\frac{U}{N k T}, \quad f=\frac{F}{N k T},
$$

where $N$ is the number of particles in the simulation volume. The thermal component of the potential energy is defined by

$$
u_{\mathrm{th}}(\kappa, \Gamma)=u(\kappa, \Gamma)-u_{\infty}(\kappa),
$$

where $u_{\infty}(\kappa)$ represents the Madelung energy (for an appropriate lattice) per particle in units of $k T$. We also define

$$
E(\kappa)=\lim _{\Gamma \rightarrow \infty} \frac{u(\kappa, \Gamma)}{\Gamma},
$$

i.e., the Madelung energy per particle in units of $Q^{2} / 4 \pi \epsilon_{0} a$, so that $u_{\infty}(\kappa)=E(\kappa) \Gamma$. Evidently $u(\kappa, \infty)=u_{\infty}(\kappa)$ in the limit of zero temperature (i.e., $\Gamma \rightarrow \infty)$. The values for the bcc and fcc Madelung energies, $E_{\mathrm{bcc}}(\kappa)$ and $E_{\mathrm{fcc}}(\kappa)$, are listed in Table I. Note that the bcc Madelung energy is smaller than the fcc Madelung energy [1] $\left[E_{\mathrm{bcc}}(\kappa)<E_{\mathrm{fcc}}(\kappa)\right]$ for $\kappa$ $<1.066$.

Since $\partial f / \partial \Gamma=u(\kappa, \Gamma) / \Gamma$, the dimensionless Helmholtz free energy for the fluid phase may be defined [3] by

$$
f_{\text {fluid }}(\kappa, \Gamma)=\int_{0}^{\Gamma} u\left(\kappa, \Gamma^{\prime}\right) \frac{d \Gamma^{\prime}}{\Gamma^{\prime}}+f_{\text {ideal }}(\Gamma) .
$$

Here the last term represents the ideal-gas contribution to the total free energy, i.e.,

$$
\begin{aligned}
f_{\text {ideal }}(\Gamma) & =\ln \left[\left(\frac{2 \pi \hbar^{2}}{m k T}\right)^{3 / 2} n\right]-1 \\
& =3 \ln \Gamma+\frac{3}{2} \ln (k T)_{\mathrm{Ry}}-1+\ln \frac{3 \sqrt{\pi}}{4},
\end{aligned}
$$

where $(k T)_{\mathrm{Ry}}$ denotes $k T$ measured in Rydberg units, $\frac{1}{2}\left(Q^{2} / 4 \pi \epsilon_{0} \hbar\right)^{2} m$, for the particles [1]. Although $f_{\text {ideal }}$ depends on $(k T)_{\text {Ry }}$ as well as $\Gamma$, we do not explicitly express the dependence on the former for the sake of simplicity.

For the solid phase, we use [3]

$$
f_{\text {solid }}(\kappa, \Gamma)=\int_{\infty}^{\Gamma}\left(u_{\text {th }}\left(\kappa, \Gamma^{\prime}\right)-\frac{3}{2}\right) \frac{d \Gamma^{\prime}}{\Gamma^{\prime}}+f_{\text {harm }}(\kappa, \Gamma),
$$

where $u_{\mathrm{th}}-\frac{3}{2}$ is the anharmonic component of the potential energy in units of $k T$. The free energy of the harmonic lattice vibrations for a given lattice may be written as

$$
f_{\text {harm }}(\kappa, \Gamma)=E(\kappa) \Gamma+\Sigma(\kappa)+\frac{9}{2} \ln \Gamma+\frac{3}{2} \ln (k T)_{\mathrm{Ry}}+\frac{3}{2} \ln \frac{3}{2},
$$

where $\Sigma(\kappa)$ denotes the harmonic entropy constant, i.e.,

$$
\Sigma(\kappa)=\lim _{N \rightarrow \infty} \frac{1}{N} \sum_{k=1}^{3 N-3} \ln \frac{\omega_{k}}{\omega_{p}} .
$$

Here the sum is taken over the $3 N-3$ normal-mode frequencies $\omega_{k}$ for the oscillation of an $N$-particle lattice. Note that the values of $E(\kappa)$ and $\Sigma(\kappa)$ depend on the chosen lattice structure. The eigenfrequencies $\omega_{k}$ of an $N$-particle Yukawa lattice may be computed by standard techniques [24], and the quantity $\Sigma(\kappa)$ can then be estimated for various $\kappa$ values by letting $N \rightarrow \infty$. Table II gives the values of $\Sigma(\kappa)$ for bcc and fcc Yukawa lattices, i.e., $\Sigma_{\text {bcc }}(\kappa)$ and $\Sigma_{\text {fcc }}(\kappa)$, obtained from lattice-dynamics calculations. Note that for $\kappa>4.76$ the bcc structure is unstable against shear in the (110) direction [20].

\section{MD SIMULATION METHOD}

MC and MD simulations can handle only a finite number of particles in the direct pairwise summation of interparticle potential energies. In order to emulate a system with an infinite number of particles, one may apply periodic boundary conditions to the simulation volume. For a cubical simulation box of side length $L$, the effective pair potential [3] under periodic boundary conditions becomes

$$
\Phi(\mathbf{r})=\phi(|\mathbf{r}|)+\sum_{\mathbf{n} \neq \mathbf{0}} \phi(|\mathbf{r}+\mathbf{n} L|) .
$$

In the above equation, $\Phi(\mathbf{r})$ represents the interaction energy of particle $i$ with particle $j$ (at separation $\mathbf{r}=\mathbf{r}_{j}-\mathbf{r}_{i}$ ) and with all periodic images of the latter. The infinite sum of $\phi$ over integer vectors $\mathbf{n}=(l, m, n)$ represents the periodic images. In our MD simulations, this periodic image potential is approximated numerically by a tensor-product spline function [25] interpolating an array of $40 \times 40 \times 40$ discrete values, 
TABLE II. Harmonic entropy constants for bcc and fcc Yukawa lattices. For $\kappa \leqslant 1.0$, see Table I of Ref. [1]. Note that for $\kappa>4.76$ the bcc structure becomes unstable against shear in the (110) direction [20].

\begin{tabular}{|c|c|c|}
\hline$\kappa$ & $\Sigma_{\mathrm{bcc}}$ & $\Sigma_{\mathrm{fcc}}$ \\
\hline 1.2 & -3.1773 & -3.1236 \\
\hline 1.4 & -3.3950 & -3.3366 \\
\hline 1.6 & -3.6350 & -3.5714 \\
\hline 1.8 & -3.8953 & -3.8259 \\
\hline 2.0 & -4.1740 & -4.0985 \\
\hline 2.2 & -4.4697 & -4.3876 \\
\hline 2.4 & -4.7805 & -4.6916 \\
\hline 2.6 & -5.1054 & -5.0095 \\
\hline 2.8 & -5.4430 & -5.3398 \\
\hline 3.0 & -5.7922 & -5.6817 \\
\hline 3.2 & -6.1522 & -6.0341 \\
\hline 3.4 & -6.5220 & -6.3962 \\
\hline 3.6 & -6.9008 & -6.7673 \\
\hline 3.8 & -7.2878 & -7.1466 \\
\hline 4.0 & -7.6826 & -7.5334 \\
\hline 4.2 & -8.0847 & -7.9273 \\
\hline 4.4 & -8.4936 & -8.3275 \\
\hline 4.6 & -8.9095 & -8.7337 \\
\hline 4.8 & & -9.1455 \\
\hline 5.0 & & -9.5625 \\
\hline
\end{tabular}

summed to high accuracy. The approximation can be efficiently evaluated in the simulations, and has a fractional deviation from the exact value of no more than $\sim 10^{-7}$. Full details of the approximation scheme may be found in Ref. [26].

The total potential energy (or "excess energy") $U$ in the simulation box with periodic boundary conditions is then given by the expression [1]

$$
\begin{aligned}
\frac{U}{N k T}= & \Gamma\left[\frac{1}{N} \sum_{j=1}^{N-1} \sum_{k=j+1}^{N} \hat{\Phi}\left(\boldsymbol{\xi}_{k}-\boldsymbol{\xi}_{j}\right)-\frac{3}{2 \kappa^{2}}-\frac{\kappa}{2}\right. \\
& \left.+\frac{1}{2} \sum_{\mathbf{n} \neq \mathbf{0}} \frac{\exp (-\kappa|\mathbf{n}| \Lambda)}{|\mathbf{n}| \Lambda}\right],
\end{aligned}
$$

where $\Lambda=L / a=(4 \pi N / 3)^{1 / 3}$ is the size of the cubical simulation volume in units of the Wigner-Seitz radius, $\boldsymbol{\xi}_{i}=\mathbf{r}_{i} / a$ is the dimensionless location of particle $i$, and $\hat{\Phi}=4 \pi \varepsilon_{0} a \Phi / Q^{2}$.

As in the earlier study [1], we employ MD simulations with the effective pair potential $\Phi(\mathbf{r})$ given by Eq. (9) to evaluate the potential energy $u$ for given values of the thermodynamic variables $\kappa$ and $\Gamma$. The equations of motion

$$
\frac{d^{2} \mathbf{r}_{i}}{d t^{2}}=-\sum_{j(\neq i)}^{N} \boldsymbol{\nabla} \Phi\left(\mathbf{r}_{i}-\mathbf{r}_{j}\right) \quad \text { for } i=1, \ldots, N
$$

are integrated, and the velocities of all particles are renormalized periodically to bring the system kinetic energy into agreement with the target $\Gamma$ value. The number of particles $N$ used for the simulations reported here are $N=686$ for a bcc and $N=500$ for a fcc lattice. These lattices are used as initial conditions, and the system is allowed to equilibrate to the desired $\Gamma$ for typically 100 time units before averaging its properties over $100<\tau<300$. Here the time unit is defined to be $\sqrt{3} \omega_{p}^{-1}$, where $\omega_{p}=\sqrt{Q^{2} n / \epsilon_{0} m}$ is the plasma frequency for the particles, so that $\tau=\omega_{p} t / \sqrt{3}$. For some large $\Gamma$ values, we allowed the the system to equilibrate initially for 300 time units. Cases that melted to a fluid state did so well before $\tau=100$.

The excess energy per particle in units of $Q^{2} / 4 \pi \epsilon_{0} a$ (i.e., $u / \Gamma$ ) obtained from the MD simulations is listed for various $\Gamma$ and $\kappa$ values in Tables III-V. For each of the runs in these tables, the initial state is either a bcc $(N=686)$ or a fcc $(N$ $=500$ ) lattice, and therefore in the solid state the structure remains in the form of the chosen lattice.

For the fluid phase, we assume that the potential energy depends on $\Gamma$ as

$$
u(\kappa, \Gamma)=a(\kappa) \Gamma+b(\kappa) \Gamma^{s}+c(\kappa)+d(\kappa) \Gamma^{-s},
$$

with $s=1 / 3$, for $\Gamma \geqslant 1$. The coefficients $a, b, c$, and $d$, which are functions of $\kappa$, are determined by fitting measured potential energies given in Table III to the above expression. The functional form of Eq. (11) has been applied to internal energy fitting of various OCP simulations [16]. The welldefined dependence of $u$ on $\Gamma$ given in Eq. (11) makes it easier to evaluate the integral in Eq. (4). Since Eq. (11) diverges as $\Gamma \rightarrow 0$, we evaluate the integral in Eq. (4) by a direct numerical quadrature for $\Gamma \leqslant 1$ :

$$
f_{\text {fluid }}(\kappa, \Gamma)=\int_{1}^{\Gamma} u\left(\kappa, \Gamma^{\prime}\right) \frac{d \Gamma^{\prime}}{\Gamma^{\prime}}+f_{1}(\kappa)+f_{\text {ideal }}(\Gamma),
$$

with

$$
f_{1}(\kappa)=\int_{0}^{1} u\left(\kappa, \Gamma^{\prime}\right) \frac{d \Gamma^{\prime}}{\Gamma^{\prime}}
$$

where Eq. (11) is used to evaluate the first integral in Eq. (12), and $f_{1}(\kappa)$ is evaluated through a Simpson-rule quadrature of the $u / \Gamma$ values given in Table VI. Note that $u / \Gamma \rightarrow$ $-\kappa / 2$ as $\Gamma \rightarrow 0$ [27]. The numerical values of $f_{1}(\kappa)$ are listed in Table VII.

For the solid phase, the following form for the thermal potential energy is assumed:

$$
u_{\mathrm{th}}(\kappa, \Gamma)=\frac{3}{2}+\frac{A_{1}(\kappa)}{\Gamma}+\frac{A_{2}(\kappa)}{\Gamma^{2}}
$$

where $\frac{3}{2}$ is the harmonic component, and the power series in $\Gamma^{-1}$ represents the anharmonic terms.

To determine the fitting parameters $a, b, c, d, A_{1}$, and $A_{2}$ for each $\kappa$ value, we fit the potential energy functional forms, Eqs. (11) and (14), to the simulation data given in Tables III-V, using least-squares fitting. The resulting coefficient values are given in Tables VIII and IX for $\kappa \geqslant 1.2$. Figure 1 presents examples of least-squares fitting to the fluid and solid phases at $\kappa=3.0$. The dotted lines represent the ranges of fitting uncertainties, which will be discussed in the next section.

For $\kappa \leqslant 1$, we assume a polynomial dependence of the normalized potential energy $u$ on $\kappa$ (i.e., a Taylor series ex- 
TABLE III. Excess energy per particle, $u / \Gamma$, obtained from MD simulations. The numbers after \pm indicate fluctuation levels. The number of simulation particles is $N=686$ for $\kappa=4.0$ and 5.0, and $N=500$ for all other $\kappa$ values. For $\kappa \leqslant 1.0$, see Table II of Ref. [3].

\begin{tabular}{|c|c|c|c|}
\hline$\Gamma$ & $\kappa=1.2$ & $\kappa=1.4$ & $\kappa=2.0$ \\
\hline 1 & $-0.797097 \pm 0.010904$ & $-0.869746 \pm 0.011675$ & $-1.110066 \pm 0.009674$ \\
\hline 2 & $-0.855439 \pm 0.009179$ & $-0.920097 \pm 0.008272$ & \\
\hline 5 & $-0.921138 \pm 0.004200$ & $-0.978642 \pm 0.004510$ & $-1.184378 \pm 0.003545$ \\
\hline 10 & $-0.958561 \pm 0.002799$ & $-1.012022 \pm 0.002587$ & $-1.208846 \pm 0.002178$ \\
\hline 20 & $-0.985481 \pm 0.001533$ & $-1.037095 \pm 0.001467$ & $-1.227788 \pm 0.001343$ \\
\hline 40 & $-1.004536 \pm 0.000893$ & $-1.055146 \pm 0.000911$ & $-1.241424 \pm 0.000743$ \\
\hline 60 & $-1.012768 \pm 0.000683$ & $-1.062759 \pm 0.000616$ & $-1.247832 \pm 0.000638$ \\
\hline 80 & $-1.017387 \pm 0.000559$ & $-1.067144 \pm 0.000495$ & $-1.251210 \pm 0.000509$ \\
\hline 100 & $-1.020683 \pm 0.000433$ & $-1.070322 \pm 0.000426$ & $-1.253666 \pm 0.000469$ \\
\hline 120 & $-1.022726 \pm 0.000427$ & $-1.072513 \pm 0.000409$ & \\
\hline 140 & $-1.024535 \pm 0.000353$ & $-1.074088 \pm 0.000329$ & \\
\hline 160 & $-1.025919 \pm 0.000310$ & $-1.075469 \pm 0.000253$ & \\
\hline 180 & $-1.027188 \pm 0.000249$ & $-1.076467 \pm 0.000301$ & \\
\hline 200 & $-1.028005 \pm 0.000250$ & $-1.077485 \pm 0.000250$ & $-1.259467 \pm 0.000268$ \\
\hline 240 & & $-1.078874 \pm 0.000204$ & \\
\hline$\Gamma$ & $\kappa=2.6$ & $\kappa=3.0$ & $\kappa=3.6$ \\
\hline 1 & $-1.377115 \pm 0.008032$ & $-1.563017 \pm 0.006990$ & $-1.847809 \pm 0.005863$ \\
\hline 5 & $-1.427355 \pm 0.003300$ & $-1.602063 \pm 0.002709$ & $-1.875608 \pm 0.002235$ \\
\hline 10 & $-1.444503 \pm 0.002193$ & $-1.615664 \pm 0.001819$ & $-1.885249 \pm 0.001503$ \\
\hline 20 & $-1.457552 \pm 0.001221$ & $-1.625862 \pm 0.001042$ & $-1.892620 \pm 0.000969$ \\
\hline 40 & $-1.467763 \pm 0.000669$ & $-1.633625 \pm 0.000660$ & $-1.898153 \pm 0.000550$ \\
\hline 60 & $-1.472134 \pm 0.000512$ & $-1.637184 \pm 0.000492$ & $-1.900557 \pm 0.000408$ \\
\hline 80 & $-1.474749 \pm 0.000426$ & $-1.639302 \pm 0.000357$ & $-1.902094 \pm 0.000293$ \\
\hline 100 & $-1.476616 \pm 0.000361$ & $-1.640850 \pm 0.000293$ & $-1.903025 \pm 0.000299$ \\
\hline 200 & $-1.480988 \pm 0.000187$ & $-1.644389 \pm 0.000195$ & $-1.905567 \pm 0.000166$ \\
\hline 400 & $-1.484027 \pm 0.000116$ & $-1.646847 \pm 0.000115$ & $-1.907295 \pm 0.000101$ \\
\hline 700 & $-1.485724 \pm 0.000076$ & $-1.648282 \pm 0.000079$ & $-1.908312 \pm 0.000057$ \\
\hline 1000 & & & $-1.908807 \pm 0.000051$ \\
\hline 2000 & & & $-1.909541 \pm 0.000025$ \\
\hline$\Gamma$ & $\kappa=4.0$ & $\kappa=4.6$ & $\kappa=5.0$ \\
\hline 1 & $-2.040283 \pm 0.004831$ & $-2.332756 \pm 0.004502$ & $-2.528765 \pm 0.003806$ \\
\hline 5 & $-2.063313 \pm 0.001730$ & $-2.350043 \pm 0.001769$ & $-2.542942 \pm 0.001500$ \\
\hline 10 & $-2.070905 \pm 0.001221$ & $-2.355369 \pm 0.001234$ & $-2.547653 \pm 0.000874$ \\
\hline 20 & $-2.077051 \pm 0.000695$ & $-2.359643 \pm 0.000679$ & $-2.551214 \pm 0.000552$ \\
\hline 40 & $-2.081242 \pm 0.000456$ & $-2.362851 \pm 0.000449$ & $-2.553848 \pm 0.000315$ \\
\hline 60 & $-2.083236 \pm 0.000332$ & $-2.364342 \pm 0.000337$ & $-2.554926 \pm 0.000240$ \\
\hline 80 & $-2.084381 \pm 0.000227$ & $-2.365221 \pm 0.000239$ & $-2.555673 \pm 0.000196$ \\
\hline 100 & $-2.085288 \pm 0.000209$ & $-2.365722 \pm 0.000205$ & $-2.556105 \pm 0.000173$ \\
\hline 200 & $-2.087226 \pm 0.000135$ & $-2.367119 \pm 0.000122$ & $-2.557248 \pm 0.000086$ \\
\hline 400 & $-2.088586 \pm 0.000082$ & $-2.368103 \pm 0.000075$ & $-2.558051 \pm 0.000052$ \\
\hline 700 & $-2.089387 \pm 0.000049$ & $-2.368670 \pm 0.000049$ & $-2.558476 \pm 0.000036$ \\
\hline 1000 & $-2.089779 \pm 0.000040$ & $-2.368941 \pm 0.000035$ & $-2.558705 \pm 0.000023$ \\
\hline 2000 & $-2.090338 \pm 0.000019$ & $-2.369329 \pm 0.000018$ & $-2.559011 \pm 0.000017$ \\
\hline 3000 & & $-2.369513 \pm 0.000015$ & $-2.559144 \pm 0.000012$ \\
\hline 4000 & & $-2.369609 \pm 0.000014$ & $-2.559223 \pm 0.000009$ \\
\hline 5000 & & $-2.369679 \pm 0.000011$ & $-2.559275 \pm 0.000009$ \\
\hline 6000 & & $-2.369727 \pm 0.000010$ & $-2.559310 \pm 0.000006$ \\
\hline 8000 & & & $-2.559366 \pm 0.000007$ \\
\hline 10000 & & & $-2.559410 \pm 0.000005$ \\
\hline
\end{tabular}


TABLE IV. Equilibrium potential energy per particle, $u / \Gamma$, for bcc solids (with $N=686$ ). The numbers after \pm indicate fluctuation levels. The energy value with an asterisk $\left(^{*}\right)$ was not used for fitting since this value clearly deviates from either fitting curve, indicating that the system is in a mixed fluid-solid state. For $\kappa \leqslant 1.0$, see Table II of Ref. [3].

\begin{tabular}{|c|c|c|c|}
\hline$\Gamma$ & $\kappa=1.2$ & $\kappa=1.4$ & $\kappa=2.0$ \\
\hline 240 & $-1.032521 * \pm 0.000184$ & & \\
\hline 300 & $-1.034044 \pm 0.000117$ & $-1.083042 \pm 0.000134$ & \\
\hline 400 & $-1.035414 \pm 0.000103$ & $-1.084449 \pm 0.000099$ & \\
\hline 500 & $-1.036214 \pm 0.000072$ & $-1.085260 \pm 0.000088$ & \\
\hline 600 & $-1.036742 \pm 0.000064$ & $-1.085788 \pm 0.000054$ & \\
\hline 700 & & & $-1.266822 \pm 0.000063$ \\
\hline 800 & $-1.037388 \pm 0.000045$ & $-1.086444 \pm 0.000042$ & \\
\hline 1000 & $-1.037774 \pm 0.000044$ & $-1.086828 \pm 0.000038$ & $-1.267497 \pm 0.000040$ \\
\hline 2000 & & & $-1.268270 \pm 0.000022$ \\
\hline$\infty$ & -1.039292 & -1.088350 & -1.269026 \\
\hline$\Gamma$ & $\kappa=2.6$ & $\kappa=3.0$ & $\kappa=3.6$ \\
\hline 1000 & $-1.487386 \pm 0.000041$ & & \\
\hline 2000 & & $-1.650380 \pm 0.000021$ & \\
\hline 3000 & $-1.488437 \pm 0.000017$ & $-1.650640 \pm 0.000016$ & \\
\hline 4000 & $-1.488563 \pm 0.000014$ & $-1.650767 \pm 0.000016$ & $-1.910240 \pm 0.000011$ \\
\hline 5000 & $-1.488639 \pm 0.000012$ & $-1.650843 \pm 0.000014$ & $-1.910317 \pm 0.000010$ \\
\hline 6000 & & $-1.650893 \pm 0.000012$ & $-1.910368 \pm 0.000010$ \\
\hline 8000 & & $-1.650956 \pm 0.000009$ & $-1.910431 \pm 0.000009$ \\
\hline \multirow[t]{9}{*}{$\infty$} & -1.488941 & -1.651144 & -1.910618 \\
\hline & $\Gamma$ & $\kappa=4.0$ & \\
\hline & 5000 & $-2.090982 \pm 0.000009$ & \\
\hline & 6000 & $-2.091033 \pm 0.000007$ & \\
\hline & 8000 & $-2.091097 \pm 0.000006$ & \\
\hline & 10000 & $-2.091134 \pm 0.000004$ & \\
\hline & 13000 & $-2.091169 \pm 0.000003$ & \\
\hline & 16000 & $-2.091190 \pm 0.000003$ & \\
\hline & $\infty$ & -2.091283 & \\
\hline
\end{tabular}

pansion about $\kappa=0$ ) and fit the function over the dual independent variables $\kappa$ and $\Gamma$ (see Ref. [1] for details).

\section{SIMULATION RESULTS AND PHASE DIAGRAM}

The $\Gamma$ values for the fluid-solid phase transition (i.e., melting or freezing), which we denote by $\Gamma_{m}$, are those at which the fluid free energy $f_{\text {fluid }}$ equals the solid free energy $f_{\text {solid }}$ for the given $\kappa$. We take the smaller of $f_{\text {bcc }}$ and $f_{\text {fcc }}$ as $f_{\text {solid }}$ for the given $\kappa$ and $\Gamma$. Similarly, the $\Gamma$ values for the bcc-fcc phase transition, which we denote by $\Gamma_{s}$, are those at which the bcc and fcc free energies intersect. The solid and fluid free energies are calculated from Eqs. (6) and (12), as discussed in the preceding section. Such calculations show that, along the fluid-solid phase transition boundary, the free energy of the bcc phase is lower than that of the fcc phase for $\kappa \lesssim 4.3$, so we use the bcc phase as the solid phase for $\kappa$ $\lesssim 4.3$ and the fcc phase for $\kappa \gtrsim 4.3$ to obtain the $\Gamma$ values for the phase transition. Tables $X$ and XI summarize the phase boundaries (i.e., $\Gamma_{m}$ and $\Gamma_{s}$ ), as functions of $\kappa$.

Some earlier studies [19-23] have used normalizations different from Eq. (2) to represent the particulate temperature $T$ and the Debye screening length $\lambda_{D}$. For example, one may use $\rho=n^{-1 / 3}$ instead of the Wigner-Seitz radius $a$ as the length unit, and define $K=\rho / \lambda_{D}$. Note then that $K=(4 \pi / 3)^{1 / 3} \kappa \approx 1.61199 \kappa$. Kremer, Robbins, and Grest [19] normalized the temperature $T$ by the typical phonon energy of the fcc Yukawa lattice according to

$$
\mathcal{T}=\frac{k T}{m \omega_{E}^{2} \rho^{2}},
$$

where $\omega_{E}$ is the Einstein frequency for the fcc Yukawa lattice, defined by

$$
\omega_{E}^{2}=\frac{2 k_{D}^{2}}{3 m} \sum_{i \neq j} \phi\left(\left|\mathbf{r}_{i}-\mathbf{r}_{j}\right|\right)
$$

with all particles situated at fcc lattice sites. It is easy to confirm [1] that the dimensionless temperature $\mathcal{T}$ is related to $\kappa$ and $\Gamma$ as

$$
\mathcal{T}=\frac{1}{\Gamma}\left(\frac{3}{4 \pi}\right)^{2 / 3}\left[\frac{2}{3} \kappa^{2} E_{\mathrm{fcc}}(\kappa)+\frac{\kappa^{3}}{2}+1\right]^{-1}
$$


TABLE V. Equilibrium potential energy per particle, $u / \Gamma$, for fcc solids (with $N=500$ ). The numbers after \pm indicate fluctuation levels. The energy value with an asterisk $(*)$ was not used for fitting since this value clearly deviates from either fitting curve, indicating that the system is in a mixed fluid-solid state. Note that $u / \Gamma \rightarrow E_{\mathrm{fcc}}(\kappa)$ as $\Gamma \rightarrow \infty$.

\begin{tabular}{|c|c|c|c|}
\hline$\Gamma$ & $\kappa=1.2$ & $\kappa=1.4$ & $\kappa=2.0$ \\
\hline 240 & $-1.032173^{*} \pm 0.000228$ & & \\
\hline 300 & $-1.034003 \pm 0.000150$ & $-1.082998 \pm 0.000165$ & \\
\hline 400 & $-1.035392 \pm 0.000110$ & $-1.084437 \pm 0.000114$ & \\
\hline 500 & $-1.036209 \pm 0.000085$ & $-1.085274 \pm 0.000091$ & \\
\hline 600 & $-1.036735 \pm 0.000071$ & $-1.085804 \pm 0.000082$ & \\
\hline 700 & & & $-1.266858 \pm 0.000068$ \\
\hline 800 & $-1.037390 \pm 0.000058$ & $-1.086460 \pm 0.000057$ & \\
\hline 1000 & $-1.037778 \pm 0.000046$ & $-1.086849 \pm 0.000049$ & $-1.267541 \pm 0.000051$ \\
\hline 2000 & & & $-1.268320 \pm 0.000031$ \\
\hline$\infty$ & -1.039302 & -1.088374 & -1.269079 \\
\hline$\Gamma$ & $\kappa=2.6$ & $\kappa=3.0$ & $\kappa=3.6$ \\
\hline 1000 & $-1.487424 \pm 0.000061$ & & \\
\hline 2000 & & $-1.650422 \pm 0.000029$ & \\
\hline 3000 & $-1.488490 \pm 0.000021$ & $-1.650685 \pm 0.000016$ & \\
\hline 4000 & $-1.488618 \pm 0.000017$ & $-1.650813 \pm 0.000010$ & $-1.910270 \pm 0.000013$ \\
\hline 5000 & $-1.488695 \pm 0.000015$ & $-1.650890 \pm 0.000011$ & $-1.910349 \pm 0.000014$ \\
\hline 6000 & & $-1.650941 \pm 0.000010$ & $-1.910400 \pm 0.000013$ \\
\hline 8000 & & & $-1.910464 \pm 0.000010$ \\
\hline$\infty$ & -1.488998 & -1.651194 & -1.910653 \\
\hline$\Gamma$ & $\kappa=4.0$ & $\kappa=4.6$ & $\kappa=5.0$ \\
\hline 5000 & $-2.091000 \pm 0.000010$ & & \\
\hline 6000 & $-2.091054 \pm 0.000011$ & & \\
\hline 8000 & $-2.091119 \pm 0.000006$ & & \\
\hline 10000 & $-2.091158 \pm 0.000004$ & $-2.369920 \pm 0.000006$ & \\
\hline 13000 & $-2.091193 \pm 0.000004$ & $-2.369956 \pm 0.000004$ & \\
\hline 16000 & $-2.091214 \pm 0.000004$ & $-2.369979 \pm 0.000003$ & \\
\hline 20000 & & $-2.369997 \pm 0.000003$ & $-2.559530 \pm 0.000003$ \\
\hline 25000 & & & $-2.559546 \pm 0.000002$ \\
\hline 30000 & & $-2.370022 \pm 0.000002$ & $-2.559556 \pm 0.000002$ \\
\hline 40000 & & $-2.370034 \pm 0.000002$ & $-2.559568 \pm 0.000003$ \\
\hline 50000 & & & $-2.559576 \pm 0.000002$ \\
\hline$\infty$ & -2.091309 & -2.370072 & -2.559606 \\
\hline
\end{tabular}

The phase-transition temperatures expressed by $\mathcal{T}$, denoted by $\mathcal{T}_{m}$ and $\mathcal{T}_{s}$, are also listed in Tables $\mathrm{X}$ and XI.

Figure 2 shows the phase diagram of Yukawa systems in the $(\kappa, \mathcal{T})$ plane. Here, filled circles indicate the fluid-bcc solid phase transition, filled squares identify the fluid-fcc solid transition (from the last two rows of Table X), and filled triangles correspond to the bcc-fcc transition (from Table XI). To smoothly fit the fluid-solid phase transition data in Fig. 2, we have used the following functions:

$$
\mathcal{T}_{m}=0.002240+0.000181 \kappa+0.000209 \kappa^{2}
$$

for $0.0 \leqslant \kappa \leqslant 1.0$,

$$
\begin{gathered}
\mathcal{T}_{m}=0.00330220-0.00031200(2.6-\kappa) \\
-0.00002336(2.6-\kappa)^{2}-0.00002764(2.6-\kappa)^{3} \\
\text { for } 1.0 \leqslant \kappa \leqslant 2.6 \\
\mathcal{T}_{m}=0.002491+0.000312 \kappa \text { for } 2.6 \leqslant \kappa \leqslant 5.0 .
\end{gathered}
$$

Equation (17) is the polynomial fit to simulation data $\mathcal{T}_{m}$ for $0.0 \leqslant \kappa \leqslant 1.4$ obtained in the earlier study [1], which we have used as the fitting curve only for $0.0 \leqslant \kappa \leqslant 1.0$ in Fig. 2 . Equation (19) is a linear least-squares fit of the $\mathcal{T}_{m}$ values in Table X over $1.2 \leqslant \kappa \leqslant 5.0$, which we have used for $2.6 \leqslant \kappa$ $\leqslant 5.0$ in Fig. 2. As a guide to the eye, these two functions are smoothly connected by the cubic polynomial given in Eq. (18) for $1.0 \leqslant \kappa \leqslant 2.6$. 
TABLE VI. Excess energy per particle, $u / \Gamma$, at small $\Gamma$ values $(N=500)$. For $\kappa<1.0$, see Table IV of Ref. [3]. Note that $u / \Gamma \rightarrow-\kappa / 2$ as $\Gamma \rightarrow 0$.

\begin{tabular}{cccccc}
\hline \hline$\Gamma$ & $\kappa=1.0$ & $\kappa=1.2$ & $\kappa=1.4$ & $\kappa=2.0$ & $\kappa=2.6$ \\
\hline 0.00 & -0.500000 & -0.600000 & -0.700000 & -1.000000 & -1.300000 \\
0.10 & -0.557792 & -0.648425 & -0.740415 & -1.025685 & -1.319127 \\
0.20 & -0.596414 & -0.680595 & -0.770037 & -1.042716 & -1.331495 \\
0.40 & -0.648070 & -0.724539 & -0.806296 & -1.067750 & -1.348344 \\
0.60 & -0.684570 & -0.757346 & -0.831754 & -1.085879 & -1.360226 \\
0.80 & -0.713870 & -0.782004 & -0.852470 & -1.099115 & -1.369705 \\
1.00 & -0.734226 & -0.797097 & -0.869746 & -1.110066 & -1.377115 \\
$\Gamma$ & $\kappa=3.0$ & $\kappa=3.6$ & $\kappa=4.0$ & $\kappa=4.6$ & $\kappa=5.0$ \\
\hline 0.00 & -1.500000 & -1.800000 & -2.000000 & -2.300000 & -2.500000 \\
0.10 & -1.517696 & -1.815104 & -2.009731 & -2.309720 & -2.509010 \\
0.20 & -1.527809 & -1.821103 & -2.018270 & -2.315081 & -2.512796 \\
0.40 & -1.540813 & -1.831725 & -2.027327 & -2.321292 & -2.520736 \\
0.60 & -1.549763 & -1.838610 & -2.033013 & -2.327181 & -2.523305 \\
0.80 & -1.558136 & -1.843731 & -2.037149 & -2.329887 & -2.525945 \\
1.00 & -1.563017 & -1.847809 & -2.040283 & -2.332756 & -2.528765 \\
\hline \hline
\end{tabular}

The bcc-fcc phase transition curve is fitted by the following functions:

$$
\begin{gathered}
\mathcal{T}_{s}=0.00096377(\kappa-1.066) \text { for } 1.066 \leqslant \kappa \leqslant 1.2 \\
\mathcal{T}_{s}=0.00195631-0.00119860(2.6-\kappa) \\
-0.00039599(2.6-\kappa)^{2}+0.00022850(2.6-\kappa)^{3} \\
\quad \text { for } 1.2 \leqslant \kappa \leqslant 2.6 \\
\mathcal{T}_{s}=0.001352(\kappa-1.066)-0.000050(\kappa-1.066)^{2} \\
\text { for } 2.6 \leqslant \kappa \leqslant 4.2755
\end{gathered}
$$

Equation (20) is a linear fit based on the quasiharmonic theory [20]. The quasiharmonic theory is known to be accu-

TABLE VII. Values of $f_{1}(\kappa)=f_{\text {fluid }}(\kappa, 1)-f_{\text {ideal }}(1)$, defined by Eq. (13).

\begin{tabular}{cc}
\hline \hline$\kappa$ & $f_{1}(\kappa)$ \\
\hline 0.00 & -0.436765 \\
0.20 & -0.449484 \\
0.40 & -0.480913 \\
0.60 & -0.528365 \\
0.80 & -0.586650 \\
1.00 & -0.654089 \\
1.20 & -0.730380 \\
1.40 & -0.810280 \\
2.00 & -1.070980 \\
2.60 & -1.350351 \\
3.00 & -1.542363 \\
3.60 & -1.832581 \\
4.00 & -2.027406 \\
4.60 & -2.322260 \\
5.00 & -2.519954 \\
\hline
\end{tabular}

rate near $\kappa=1.066$ (where $E_{\mathrm{bcc}}=E_{\mathrm{fcc}}$ ), so we have used Eq. (20) as the fitting curve for $1.066 \leqslant \kappa \leqslant 1.2$. Equation (22) is a quadratic least-squares fit to the $\mathcal{T}_{s}$ values for $1.066 \leqslant \kappa$ $\leqslant 4.0$ given in Table XI. Although data near $\kappa=1.066$ were used to obtain Eq. (22), it does not reproduce the $\mathcal{T}_{m}$ values near $\kappa=1.066$ very well. Therefore, in Fig. 2, we use Eq. (22) as the fitting curve for $\kappa \geqslant 2.6$ only. The cubic polynomial given in Eq. (21) is used to smoothly connect these two functions over $1.2 \leqslant \kappa \leqslant 2.6$. The point where the three phases (fluid, bcc, and fcc lattices) meet-i.e., the triple point - is the intersection of Eqs. (19) and (22), which is given as $\kappa=4.28(K=6.90)$ and $\mathcal{T}=0.0038$.

It is not easy to accurately estimate the magnitude of the errors in the phase-transition boundary curves. There may be several possible sources of uncertainties. In the case of large $\Gamma$, for example, the potential energy $u$ is very close to the Madelung energy $u_{\infty}$ and the numerical value for the difference $u_{\mathrm{th}}=u-u_{\infty}$, which is used to determine the phase diagram, has fewer meaningful digits. Furthermore, if $\kappa$ and $\Gamma$ are large (and therefore the interparticle interaction is weak and the system has a low thermal energy), it takes longer (in terms of the time unit $\sqrt{3} \omega_{p}^{-1}$ ) for the system to attain ther-

TABLE VIII. Fluid fitting parameters, $a, b, c$, and $d$ defined by Eq. (11). For $\kappa \leqslant 1.0$, see Ref. [3].

\begin{tabular}{ccccc}
\hline \hline$\kappa$ & $a$ & $b$ & $c$ & $d$ \\
\hline 1.2 & -1.041816 & 0.522733 & -0.305649 & 0.026740 \\
1.4 & -1.090801 & 0.514325 & -0.344195 & 0.049258 \\
2.0 & -1.270571 & 0.442193 & -0.382900 & 0.100506 \\
2.6 & -1.489806 & 0.366308 & -0.411566 & 0.159826 \\
3.0 & -1.651703 & 0.312503 & -0.394913 & 0.173963 \\
3.6 & -1.910871 & 0.239251 & -0.362000 & 0.195448 \\
4.0 & -2.091363 & 0.182517 & -0.257154 & 0.131096 \\
4.6 & -2.370109 & 0.139276 & -0.232476 & 0.142315 \\
5.0 & -2.559633 & 0.115580 & -0.215437 & 0.149102 \\
\hline \hline
\end{tabular}




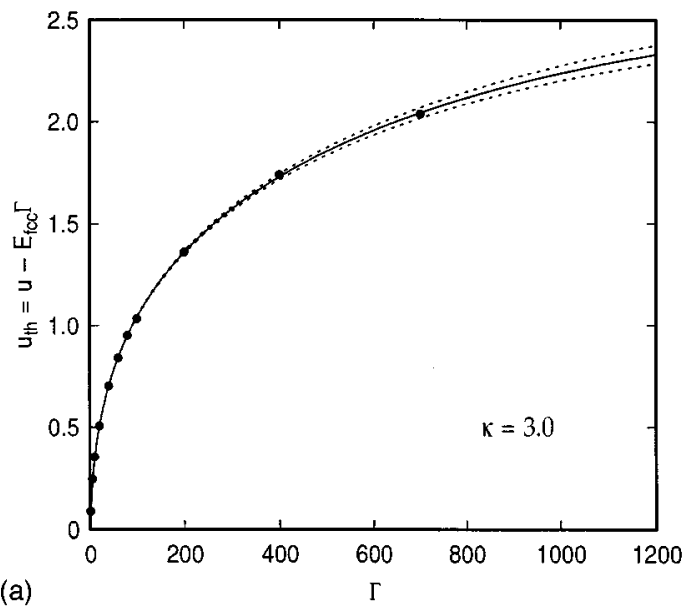

(a)

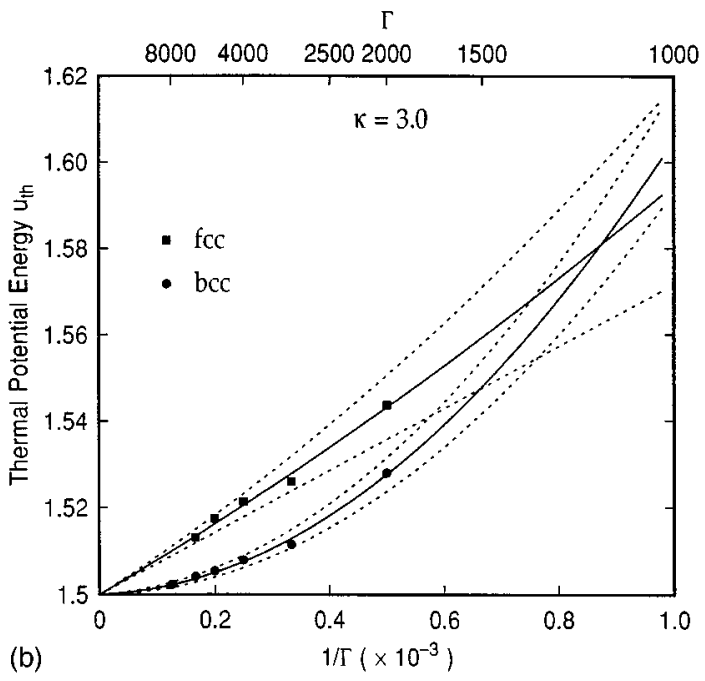

FIG. 1. (a) The thermal potential energy in the fluid phase, defined by $u_{\mathrm{th}}=u-E_{\mathrm{fcc}} \Gamma$, at $\kappa=3.0$. The filled circles are data obtained from Table III. The solid line is the least-squares fit to the data; the dotted lines represent the range of uncertainties due to the coefficient $a=-1.651703 \pm 0.000037$. (b) The thermal potential energies at $\kappa=3.0$, defined by $u_{\mathrm{th}}=u-E_{\mathrm{bcc}} \Gamma$ for the bcc lattice and $u_{\mathrm{th}}=u-E_{\mathrm{fcc}} \Gamma$ for the fcc lattice. The filled circles are bcc data obtained from Table IV, and the filled squares are fcc data obtained from Table V. The solid lines are the least-squares fits to these data. The dotted lines represent the ranges of uncertainties due to the coefficients $A_{1}=5.344 \pm 3.462, A_{2}=99742.3 \pm 8643.8$ for the bcc lattice, and $A_{1}=77.822 \pm 6.653, A_{2}=16822.5 \pm 16374.6$ for the fcc lattice.

mal equilibrium. Consequently, the measured energy data are prone to errors due to numerical averaging over finite time intervals.

Therefore we take a pragmatic approach to estimating the uncertainties associated with the phase boundaries. Assuming the measured energy values have uniform errors given by the square root of the sample variance, one can estimate the uncertainties of the fitting parameters $[18,28]$. For example, for the bcc solid phase at $\kappa=3.0$, we obtain $A_{1}=5.344$ \pm 3.462 and $A_{2}=99742.3 \pm 8643.8$, the numbers after \pm representing the range of the uncertainties. In the fluid phase, it follows from Eq. (11) that the energy value $u$ is most sensitive to variation in the coefficient $a(\kappa)$ for large $\Gamma$. Its uncertainty under the same assumption is
TABLE IX. Solid fitting parameters $A_{1}$ and $A_{2}$ for bcc and fcc Yukawa lattices defined by Eq. (14). For $\kappa \leqslant 1.0$, see Ref. [3].

\begin{tabular}{rrrrr}
\hline \hline$\kappa$ & \multicolumn{1}{c}{$A_{1}^{\text {bcc }}$} & \multicolumn{1}{c}{$A_{2}^{\text {bcc }}$} & \multicolumn{1}{c}{$A_{1}^{\text {fcc }}$} & \multicolumn{1}{c}{$A_{2}^{\text {fcc }}$} \\
\hline 1.2 & 15.42 & 2042.56 & 21.13 & 1712.24 \\
1.4 & 16.12 & 3398.78 & 17.87 & 4735.20 \\
2.0 & 23.53 & 4526.98 & 37.68 & 414.70 \\
2.6 & 30.16 & 24377.67 & 67.44 & 5735.30 \\
3.0 & 5.34 & 99742.33 & 77.82 & 16822.48 \\
3.6 & -45.39 & 392246.46 & 91.35 & 151465.97 \\
4.0 & -175.68 & 1067933.16 & 25.63 & 904495.83 \\
4.6 & & & -114.88 & 2828867.93 \\
5.0 & & & -1069.10 & 27561540.63 \\
\hline \hline
\end{tabular}

$a=-1.651703 \pm 0.000037$ [see Fig. 1(a)].

If we choose the most probable values of $a, b, c, d$ at $\kappa=3.0$, as given in Table VIII, and vary $A_{1}$ and $A_{2}$ as indicated above, the range of uncertainties for the melting point becomes $3.468 \times 10^{-3}<\mathcal{T}_{m}<3.529 \times 10^{-3}$ at $\kappa=3.0$. On the other hand, if we use the most probable values $A_{1}=5.344$ and $A_{2}=99742.3$ and vary $a$ in the range -1.651703 \pm 0.000037 , the uncertainty in the melting point at $\kappa=3.0$ becomes $3.274 \times 10^{-3}<\mathcal{T}_{m}<3.719 \times 10^{-3}$. The error bar on the melting curve in Fig. 2 represents the latter range of uncertainty - the larger of the two.

Similarly, for the solid phases at $\kappa=3.0$, we obtain $A_{1}$ $=5.344 \pm 3.462, A_{2}=99742.3 \pm 8643.8$ for the bcc lattice and $A_{1}=77.822 \pm 6.653, A_{2}=16822.5 \pm 16374.6$ for the fcc lattice [see Fig. 1(b)]. If we choose the most probable values of $A_{1}$ and $A_{2}$ given in Table IX for the bcc phase and vary $A_{1}$ and $A_{2}$ for the fcc phase, and vice versa, the range of $\mathcal{T}_{s}$ at $\kappa=3.0$ is found to be $2.322 \times 10^{-3}<\mathcal{T}_{s}<2.901 \times 10^{-3}$. The error bar on the bcc-fcc phase transition curve in Fig. 2 represents this range.

Figure 3 shows the same data as Fig. 2, plotted in the $(\kappa, \Gamma)$ plane. The phase boundaries $\Gamma_{m}$ and $\Gamma_{s}$ are also converted from $\mathcal{T}$ to $\Gamma$, using Eq. (16) and the fitting curves employed in Fig. 2. The errors at $\kappa=3.0$ in Fig. 2 are also

TABLE $X$. The fluid-solid phase-transition values $\Gamma$ and $\mathcal{T}$. Note that the solid phase at the phase boundary is bcc for $\kappa \leqslant 4.0$ and fcc for $\kappa \geqslant 4$.6 in this table. The normalized temperature $\mathcal{T}$ is defined by Eq. (15).

\begin{tabular}{ccc}
\hline \hline$\kappa$ & $\Gamma_{m}$ & $\mathcal{T}_{m}$ \\
\hline 0.0 & 171.8 & $2.240 \times 10^{-3}$ \\
0.2 & 173.5 & $2.267 \times 10^{-3}$ \\
0.4 & 178.6 & $2.332 \times 10^{-3}$ \\
0.6 & 187.1 & $2.425 \times 10^{-3}$ \\
0.8 & 199.6 & $2.535 \times 10^{-3}$ \\
1.0 & 217.4 & $2.647 \times 10^{-3}$ \\
1.2 & 243.3 & $2.736 \times 10^{-3}$ \\
1.4 & 268.8 & $2.907 \times 10^{-3}$ \\
2.0 & 440.1 & $3.095 \times 10^{-3}$ \\
2.6 & 758.9 & $3.420 \times 10^{-3}$ \\
3.0 & 1185 & $3.498 \times 10^{-3}$ \\
3.6 & 2378 & $3.682 \times 10^{-3}$ \\
4.0 & 3837 & $3.851 \times 10^{-3}$ \\
4.6 & 8609 & $3.881 \times 10^{-3}$ \\
5.0 & $1.506 \times 10^{4}$ & $3.888 \times 10^{-3}$ \\
\hline \hline
\end{tabular}


TABLE XI. Transition values of $\Gamma$ and $\mathcal{T}$ at the bcc-fcc phase boundary. The normalized temperature $\mathcal{T}$ is defined by Eq. (15).

\begin{tabular}{lcl}
\hline \hline$\kappa$ & $\Gamma_{s}$ & \multicolumn{1}{c}{$\mathcal{T}_{s}$} \\
\hline 1.066 & $\infty$ & 0.000 \\
1.2 & 5070 & $1.313 \times 10^{-4}$ \\
1.4 & 2325 & $3.361 \times 10^{-4}$ \\
2.0 & 1228 & $1.109 \times 10^{-4}$ \\
2.6 & 1273 & $2.040 \times 10^{-4}$ \\
3.0 & 1634 & $2.537 \times 10^{-4}$ \\
3.6 & 2884 & $3.036 \times 10^{-4}$ \\
4.0 & 4185 & $3.531 \times 10^{-4}$ \\
\hline
\end{tabular}

plotted in Fig. 3; these errors are less prominent due to the logarithmic scale for $\Gamma$. The triple point is given by $\Gamma=5.6$ $\times 10^{3}$ at $\kappa=4.28(K=6.90)$. Both the fluid-solid and bcc-fcc phase transitions are of first order $[1,20,29]$.

\section{COMPARISON WITH EARLIER WORK}

In Fig. 4, we compare our MD simulation results with those from earlier MD and MC simulations, based on different methods [19-23]. These earlier MD and MC simulations do not include the infinite sum for periodic boundary conditions-i.e., the second term in Eq. (9)—and are thus valid only in the large $\kappa$ regime (i.e., $\kappa \gtrsim 1$ ). In Fig. 4 , the linear fit given by Eq. (19) is extrapolated to $\kappa=8.0$. The filled marks and solid lines are the same as those used in Fig. 2. The crosses, together with the error bars, are the fluidsolid phase boundary points obtained by Meijer and Frenkel [21]. These values were obtained from a modified FrenkelLadd lattice-coupling method [30] and MC simulation for systems of 256 or fewer particles. The error bars show the statistical errors. The open rectangles, triangles, and diamonds indicate stable fluid, bcc, and fcc states, respectively,

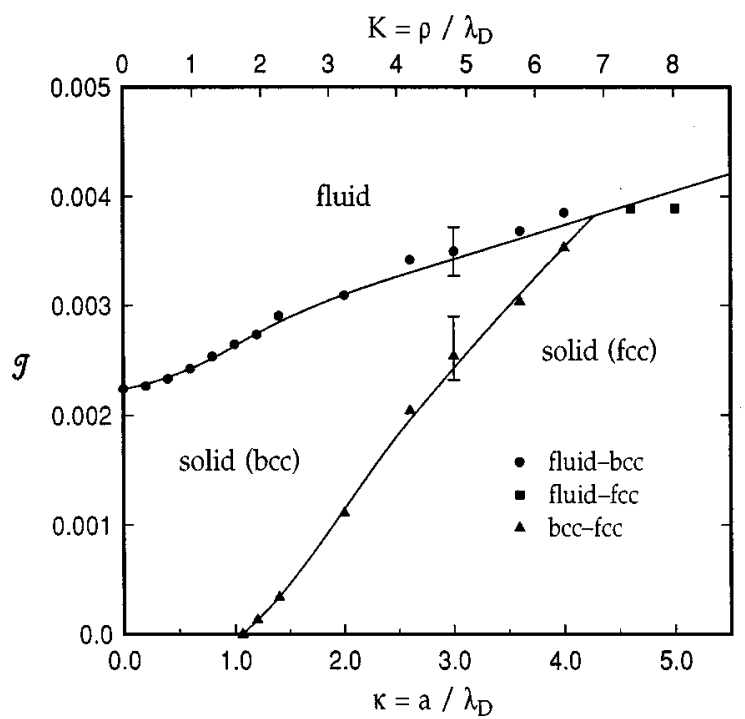

FIG. 2. Phase diagram of Yukawa systems in the $(\kappa, \mathcal{T})$ plane. The filled circles are fluid-bcc phase boundary points $(\kappa<4.3)$, the filled squares are fluid-fcc phase boundary points $(\kappa>4.3)$, and the filled triangles are bcc-fcc phase boundary points (see Tables $\mathrm{X}$ and $\mathrm{XI}$ ). The solid lines represent the curves fitted to these data points.

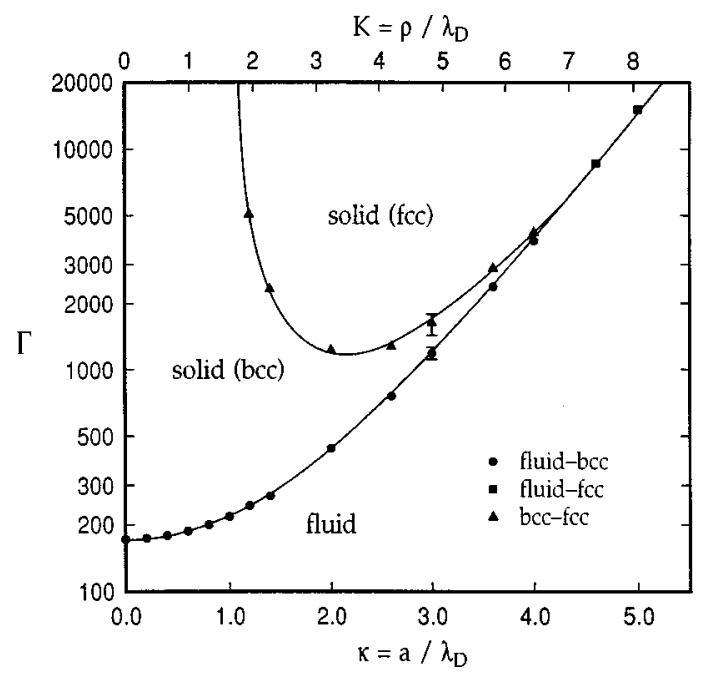

FIG. 3. Phase diagram of Yukawa systems in the $(\kappa, \Gamma)$ plane. The filled circles and solid lines are the same as those used in Fig. 2, converted to $\Gamma$ from $\mathcal{T}$ through Eq. (16).

obtained by Stevens and Robbins [22]. These authors used MD simulations and observed the time evolution of the state, starting from fluid-solid coexistent initial conditions (phenomenological melting tests) — the number of particles used in their simulations are 432 for bcc lattices and 500 for fcc lattices.

The bcc-fcc phase boundary obtained from latticedynamics calculations (quasiharmonic theory) by Robbins,

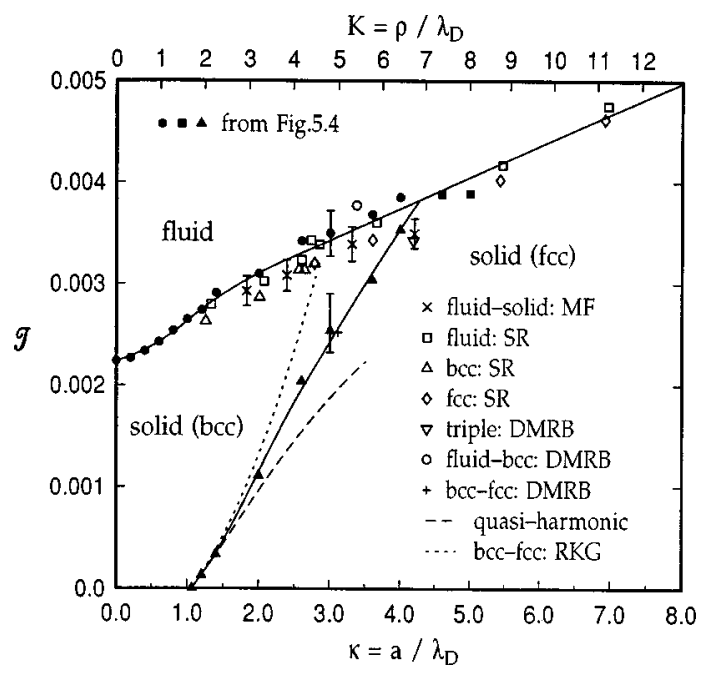

FIG. 4. Phase diagram of Yukawa systems in the $(\kappa, \mathcal{T})$ plane. The filled symbols and solid lines are from Fig. 2. The crosses $(\times)$ with error bars are fluid-solid phase boundary points obtained by Meijer and Frenkel [21]. The open rectangles $(\square)$, triangles $(\triangle)$, and diamonds $(\diamond)$ indicate fluid, bcc, and fcc states, respectively, obtained by Stevens and Robbins [22]. The open circle $(\bigcirc)$, plus $(+)$, and open inverted triangle $(\nabla)$ are a fluid-bcc boundary point, a bcc-fcc phase boundary point, and the triple point, respectively, obtained by DuPont et al. [23]. The dashed line is the bcc-fcc phase boundary obtained by Robbins, Kremer, and Grest [20], based on the quasiharmonic theory (lattice-dynamics calculations). The dotted line is also a bcc-fcc phase boundary obtained by these authors [20], based on MD simulations and the energy-distribution-function method [20,31]. 
Kremer, and Grest [20] is plotted as the dashed line in Fig. 4. The dotted line is the bcc-fcc phase boundary, also obtained by Robbins, Kremer, and Grest [20], based on MD simulations and the energy-distribution-function method [20,31]. Using this bcc-fcc phase boundary, Stevens and Robbins estimated the triple point-i.e., the boundary point of fluid, bcc, and fcc phases-as $\kappa=2.85$ and $\mathcal{T}=0.0032$. This triple point is located at much smaller $\kappa$ than that obtained in the present study.

DuPont, Moulinasse, Ryckaert, and Baus [23] used MC simulation and the Frenkel-Ladd lattice-coupling method [32] to evaluate solid free energies. Using the free energies of the fluid phase obtained by Meijer and Frenkel [21], DuPont et al. obtained a fluid-bcc boundary point, a bcc-fcc phase boundary point, and the triple point, denoted, respectively, by the open circle, plus, and open inverted triangle, in Fig. 4. This triple point-at $\kappa=4.19(K=6.75)$ and $\mathcal{T}$ $=0.0034\left(\Gamma=5.6 \times 10^{3}\right)$-is close to the one obtained in the present study, namely, $\kappa=4.28(K=6.90)$ and $\mathcal{T}=0.0038$ $\left(\Gamma=5.6 \times 10^{3}\right)$.

It is interesting to note that the triple-point $\kappa$ and $\Gamma$ values obtained by DuPont et al. and in the present study are very close (within $2 \%$ ), although the $\mathcal{T}$ values differ by over $10 \%$. This is because $\mathcal{T}$ is a sensitive function of $\kappa$ when $\kappa \gtrsim 1.0$, as shown below. From Eq. (16), one can write

$$
\frac{d \mathcal{T}}{\mathcal{T}}=-\frac{d \Gamma}{\Gamma}+\frac{\kappa}{\mathcal{T}} \frac{\partial \mathcal{T}}{\partial \kappa} \frac{d \kappa}{\kappa} .
$$

It follows from Eq. (16) that the coefficient of the second term $(\kappa / \mathcal{T})(\partial \mathcal{T} / \partial \kappa)$ above depends only on $\kappa$, and not $\Gamma$. The values of this coefficient are 2.02, 5.32, 8.88, and 12.5 at $\kappa=2.0,4.0,6.0$, and 8.0, respectively. For example, if $\kappa$ $=4.0$, a $2 \%$ error in the $\kappa$ value results in more than $10 \%$ error in the corresponding $\mathcal{T}$ value for a given $\Gamma$.

The fluid-solid phase-transition temperatures obtained in this study are systematically higher (by about $5 \%$ in $\mathcal{T}$ ) than those obtained by Meijer and Frenkel. With their modified Frenkel-Ladd lattice-coupling method [30], Meijer and Frenkel obtained the Gibbs free energy by integrating a polynomial fit to the density-pressure data obtained from MC simulations. In addition to this different methodology, other factors may have contributed to the systematic discrepancy. First, the MC simulations by Meijer and Frenkel employed relatively small numbers of particles $(N \leqslant 256)$. Second, Meijer and Frenkel assumed that the solid phase at their data points of $\kappa=3.30(K=5.33)$ and $\kappa=4.20(K=6.77)$ is fcc. However, our simulations, as well as those by DuPont et al. [23], indicate that this phase is actually bcc.

The stable fluid phase data presented by Stevens and Robbins, which are considered to give an upper bound of the fluid-solid transition phase, lie more or less on or above our fitted fluid-solid phase boundary, suggesting good agreement with our data. Only two data points given by Stevens and Robbins-those at $\kappa=2.067$ and $2.597 \quad(K=3.332$ and 4.186) - are slightly lower than our fitted phase-transition curve. These two data are obtained from MD simulations of a system of 432 particles, with the potential truncated at a radius equal to $3 \rho$, whereas we have used MD simulations of 686-particle systems for bcc lattices and 500-particle systems for fluid phases with no potential truncation. To determine the stable phase, Stevens and Robbins ran MD simulations starting from a two-phase state (equally divided fluid and solid phases) and observed its time evolution. If the difference between the free energies of the two phases is very small, which is the case near the transition point, the evolution of the MD simulation may be sensitively dependent on the shape of the simulation box, number of particles, initial perturbations, and the potential truncation radius.

The two fluid-solid boundary points obtained by DuPont, Moulinasse, Ryckaert, and Baus [23] [denoted by the open circle and open inverted triangle in Fig. 4; the latter is also the triple point] seem rather scattered if one believes that the melting temperature $\mathcal{T}_{m}$ increases linearly with $\kappa$. In the lattice-coupling calculations by DuPont et al., the free energy is obtained by integrating the energy along an isotherm. Hence the actual temperature (or equivalently $\Gamma$ ) is fixed, and $\kappa$ is computed for each phase boundary point [which is opposite to our method: we fix $\kappa$ values and determine corresponding phase-transition temperatures (or $\Gamma$ values)]. As discussed above, however, small errors in $\kappa$ can result in large $\mathcal{T}$ errors. Indeed, DuPont et al. obtain $\Gamma=1.7 \times 10^{3}$ and $5.6 \times 10^{3}$ at $\kappa=3.38$ and 4.19 as fluid-solid boundary points. Our $\kappa$ estimates on the fluid-solid boundary [from Eq. (19) converted to $\Gamma]$ for $\Gamma=1.7 \times 10^{3}$ and $5.6 \times 10^{3}$ are $\kappa=3.30$ and 4.28 , which are within $2.5 \%$ of the $\kappa$ values estimated by DuPont et al. This small difference in $\kappa$ incurs a discrepancy of about $10 \%$ in $\mathcal{T}_{m}$ at the triple point.

The bcc-fcc phase boundary point obtained by DuPont et al. [23] [denoted by a plus in Fig. 4] is in excellent agreement with the bcc-fcc phase boundary curve estimated in this study, while the bcc-fcc phase-transition temperatures obtained by Robbins, Kremer, and Grest [20] are much higher (the dotted line in Fig. 4). Consequently, the triple point suggested by Stevens and Robbins [22] is located at much smaller $\kappa$ than that obtained in this study, as previously noted. Robbins, Kremer, and Grest used the energydistribution-function method $[20,31]$ to obtain the freeenergy difference between fcc and bcc phases for given $\kappa$ and $\mathcal{T}$. To determine small differences between the free energies near the bcc-fcc boundary, one needs accurate statistics for a sampling of the energy histogram in this method. It is not clear from Ref. [20] that the statistics were adequate for an accurate estimation of these values. One example given in Ref. [20] shows that the difference in the free energies at $\kappa=3.05(K=4.92)$ and $\mathcal{T}=2.24 \times 10^{-3} \quad(\Gamma=1.97$ $\left.\times 10^{3}\right)$ is $0.03 k_{B} T$. Our MD calculations show, however, that the difference is $0.014 k_{B} T$ at $\kappa=3.00$ (note the slight difference in $\kappa$ ) and $\mathcal{T}=2.24 \times 10^{-3}$, which is different by a factor of about 2. Since the measured potential energies for $\Gamma \gtrsim 2000$ give an excellent fit to the quadratic form in Eq. (14), the error in our free-energy estimate due to the extrapolation of Eq. (14) at this position in the phase space (i.e., $\kappa$ $=3.00$ and $\mathcal{T}=2.24 \times 10^{-3}$ ) is expected to be very small.

Stevens and Robbins [22] showed that their MD simulation starting from a mixed state of fluid and fcc phases evolved to the fcc phase at $\kappa=3.604$ and $\mathcal{T}=3.429 \times 10^{-3}$, which is indicated as an open diamond in Fig. 4. However, they do not seem to claim that the bcc phase is actually more unstable than the fcc phase at this point. (At $\kappa=2.779$ and $\mathcal{T}=3.198 \times 10^{-3}$, the authors of Ref. [22] show that two runs converge to different lattices, suggesting that both the bcc 
and fcc phases are stable.) It is not clear from Ref. [22] that their phenomenological melting test can distinguish such small differences in the free energy near the phase boundary.

To summarize, we believe that the triple point obtained in this study - which is close to the one obtained by DuPont et al.-is more accurate than that suggested by Stevens and Robbins. It may be of interest to compare the different methods mentioned above, using data from the same MD or MC simulations. In this manner, one could ascertain whether the discrepancy arises from differences in the methodologies of evaluating free energies or from the intrinsic accuracy of the simulation data. Figure 5 plots the same data as for Fig. 4 , in the $(\kappa, \Gamma)$ plane. The error bars are omitted in Fig. 5 for simplicity. With this logarithmic scale for $\Gamma$, the differences among the data of the various authors are hardly discernible.

\section{CONCLUSION}

We have obtained the fluid-solid phase boundary of the Yukawa system for $\kappa \leqslant 5$, including the weakly screened regime $0 \leqslant \kappa \leqslant 1$ ( $\kappa$ is the ratio of the Wigner-Seitz radius to the Debye length). This phase transition is of first order. Unlike earlier MD or MC simulations [19-23], in which interactions were computed by pairwise summation over particles within some cutoff radius, our MD simulations use interparticle potentials summed over all particle pairs, including periodic images of particles residing in the cubical simulation box. Thus long-range particle interactions are accurately accounted for over the entire range of $\kappa$ values. For strongly screened Yukawa systems $(\kappa \gtrsim 1)$, the fluid-solid phase-transition curve obtained here is in good agreement with those of the earlier studies.

We have also estimated the bcc-fcc phase boundary by the MD simulation method. This phase transition is also first

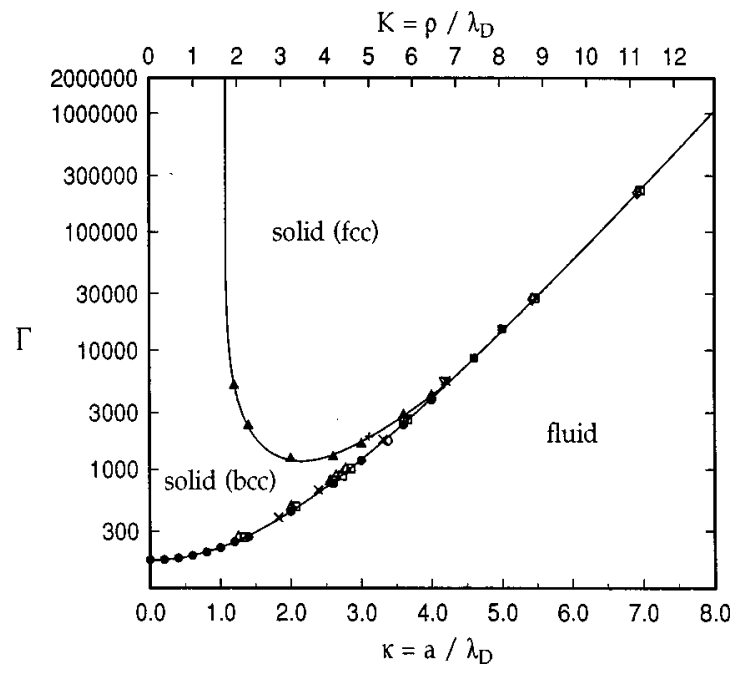

FIG. 5. Phase diagram of Yukawa systems in the $(\kappa, \Gamma)$ plane. All symbols and lines are the same as in Fig. 2, converted from $\mathcal{T}$ to $\Gamma$ through Eq. (16).

order. The transition temperatures $\mathcal{T}_{s}$ obtained are in excellent agreement with the results of quasiharmonic theory [20] near $\kappa=1.066$, the zero-temperature bcc-fcc transition point. The bcc-fcc phase-transition point for a larger $\kappa$ obtained in the recent study by DuPont, Moulinasse, Ryckaert, and Baus [23] is also in excellent agreement with our present results. The triple point (i.e., fluid-bcc-fcc phase boundary) is estimated to be $\kappa=4.28 \quad(K=6.90)$ and $\mathcal{T}=0.0038 \quad(\Gamma$ $\left.=5.6 \times 10^{3}\right)$, close to the one obtained by DuPont et al. [23]. We believe that the phase diagram presented here is the most accurate one currently available [33-35].
[1] S. Hamaguchi, R. T. Farouki, and D. H. E. Dubin, J. Chem. Phys. 105, 7641 (1996).

[2] S. Hamaguchi and R. T. Farouki, J. Chem. Phys. 101, 9876 (1994).

[3] R. T. Farouki and S. Hamaguchi, J. Chem. Phys. 101, 9885 (1994).

[4] H. Ikezi, Phys. Fluids 29, 1764 (1986).

[5] R. T. Farouki and S. Hamaguchi, Appl. Phys. Lett. 61, 2973 (1992).

[6] Y. Hayashi and K. Tachibana, Jpn. J. Appl. Phys., Part 2 33, L804 (1994).

[7] H. Thomas, G. E. Morfill, V. Demmel, J. Goree, B. Feuerbacher, and D. Möhlmann, Phys. Rev. Lett. 73, 652 (1994).

[8] J. H. Chu and I. Lin, Physica A 205, 183 (1994).

[9] J. H. Chu and I. Lin, Phys. Rev. Lett. 72, 4009 (1994).

[10] Th. Trottenberg, A. Melzer, and A. Piel, Plasma Sources Sci. Technol. 4, 450 (1995).

[11] Ordering and Organization in Ionic Solutions, edited by N. Ise and I. Sogami (World Scientific, Singapore, 1988).

[12] S. G. Brush, H. L. Sahlin, and E. Teller, J. Chem. Phys. 45, 2102 (1966).

[13] J.-P. Hansen, Phys. Rev. A 8, 3096 (1973).

[14] H. E. DeWitt, Phys. Rev. A 14, 1290 (1976).
[15] M. Baus and J.-P. Hansen, Phys. Rep. 59, 1 (1980).

[16] G. S. Stringfellow, H. E. DeWitt, and W. L. Slattery, Phys. Rev. A 41, 1105 (1990).

[17] Strongly Coupled Plasma Physics, edited by F. J. Rogers and H. E. DeWitt (Plenum, New York, 1986).

[18] R. T. Farouki and S. Hamaguchi, Phys. Rev. E 47, 4330 (1993).

[19] K. Kremer, M. O. Robbins, and G. S. Grest, Phys. Rev. Lett. 57, 2694 (1986).

[20] M. O. Robbins, K. Kremer, and G. S. Grest, J. Chem. Phys. 88, 3286 (1988).

[21] E. J. Meijer and D. Frenkel, J. Chem. Phys. 94, 2269 (1991).

[22] M. J. Stevens and M. O. Robbins, J. Chem. Phys. 98, 2319 (1993).

[23] G. DuPont, S. Moulinasse, J. P. Ryckaert, and M. Baus, Mol. Phys. 79, 453 (1993).

[24] A. A. Maradudin, E. W. Montroll, G. H. Weiss, and I. P. Ipatova, Theory of Lattice Dynamics in the Harmonic Approximation, 2nd ed. (Academic, New York, 1971).

[25] C. de Boor, A Practical Guide to Splines (Springer-Verlag, New York, 1978).

[26] R. T. Farouki and S. Hamaguchi, J. Comput. Phys. 115, 276 (1994). 
[27] S. Hamaguchi and R. T. Farouki, Phys. Rev. E 49, 4430 (1994).

[28] P. R. Bevington, Data Reduction and Error Analysis for the Physical Sciences (McGraw-Hill, New York, 1969).

[29] Y. Rosenfeld, J. Chem. Phys. 103, 9800 (1995).

[30] E. J. Meijer, D. Frenkel, R. LaSar, and A. J. C. Ladd, J. Chem. Phys. 92, 7570 (1990).
[31] A. Rahman and G. Jacucci, Nuovo Cimento D 4, 357 (1984).

[32] D. Frenkel and A. J. C. Ladd, J. Chem. Phys. 81, 3188 (1984).

[33] D. H. E. Dubin and H. E. DeWitt, Phys. Rev. B 49, 3043 (1994).

[34] D. H. E. Dubin, Phys. Rev. A 42, 4972 (1990).

[35] H. Nagara, Y. Nagata, and T. Nakamura, Phys. Rev. A 36, 1859 (1987). 